\title{
How to get an article into NJR
}

Dr. Joshi BR

NJR, the leading Nepalese journal for general radiology, is one of the major supporting tools of NRA. Due to the very limited space in all scientific journals, only the best and most innovative manuscripts can be published. Originality should ideally be the core element of a manuscript. It also helps if the results are likely to influence clinical practice. Another useful tip for authors is to keep as strictly as possible to the given manuscript structure. The specific aim of the study should be defined as sharply and precisely as possible. The conclusions should be clearly linked to the aims. Blinding of the main document should strictly be performed . As an additional new requirement, DOI should be obtained for the manuscript and mentioned appropriately. In addition, ethical guidelines require that informed consent be obtained for radiological research and for all prospective trials. The guidelines are present in NepJOL website.

Manuscripts should be prepared in accordance with "Uniform requirements for manuscripts to Biomedical Journals” JAMA 1993; 169 (17): 2282-2286

\section{Types of manuscripts and word limits}

- Original Article: Randomized controlled trials, interventional studies, studies of screening and diagnostic test, outcome studies, cost effectiveness analysis, case control series and surveys with high response rate. Up to 2500 words excluding references (up to 30 ) and abstract (up to 250 words).

- Review Article: Systematic critical assessments of literature and data sources. Up to 3000 words excluding references $(>50$ and $<100)$ and abstract $(250$ words).

- Case Report: New/ interesting/very rare cases with clinical significance or implications can be reported. Up to 1000 words excluding references (up to 10) and abstract (up to 100 words), up to three photographs.

- Letter to Editor: Should be short, decisive observation. They should not be preliminary observations that need a later paper for validation. Up to 4000 words and 5 references.

- Pictorial Assay: Should be self explanatory photographs with legends suggesting a case report.

- Limits for number of images and tables: For all the above mentioned categories, the number of images and tables should not be more than one per 500 words.

The accuracy of references is the responsibility of the authors. The list should only include works that are cited in the text. References should be numbered in the order in which they appear in the text. Digital object identifier [DOI] of the cited literature should be added at the end of the reference where available. 


\section{Articles in the Journals}

Standard journal article (list all authors, but if the number exceeds six, give three followed by et al.):

You CH, Lee KY, Chey RY, Menguy R. Electrogastrographic study of patients with unexplained nausea, bloating and vomiting. Gastroenterology 1980 Aug;79(2):311-314.

\section{Books and Monographs}

Personal author(s)

Colson JH, Armour WJ. Sports injuries and their treatment. 2nd rev. ed. London: S. Paul, 1986.

Editor(s), compiler as author:

Diener HC, Wilkinson M, editors. Drug-induced headache. New York: Springer-Verlag, 1988.

The next step is to create a cover letter to the editor-in -chief mentioning type of manuscript and its significance. Authorship document should contain the principle author, co-authors, corresponding authors and their affiliated institutions. All persons who made substantial contributions but who are not involved in the manuscript preparation are named in the acknowledgement. The corresponding author is responsible to return the galley proof of the manuscript within three days. Declaration document should mention that the research was original and has not been published or not under consideration for publication elsewhere, no conflict of interest and copyright transfer to NJR must be mentioned.

Informed consent and ethical approval letter should be taken before starting the research. These documents may be sent by email with the manuscript for preliminary approval. After a general check for completeness and correct referencing from the editorial board and a first assessment from the editor -in-chief, the manuscript is sent to reviewers. The names of authors are not revealed to the reviewers and vice versa. Reviewers thoroughly examine the manuscript and send any suggestions for possible changes. After this process, the editorial office contacts the author and presents the final decision. 